\title{
Constraint on Steady State Output Imposed by Zeros at $s=0$ and Servo Synthesis Using
} Unstable Weight

\author{
Sergio B. Villas-BoAs*, Kang-Zhi LiU* and Tsutomu MitA**
}

\begin{abstract}
When an MIMO plant has zeros at $s=0$, it will be shown that this imposes a constraint on the steady state output of the plant. In the control design using unstable weight, the structure of unstable weight can not be selected freely in this case. The required structure is exposed in this note. We then propose a particular generalized plant structure for an optimal servo design such as the one using $\mathcal{H}_{\infty}$ control.
\end{abstract}

Key Words: plant with zero at $s=0$, MIMO systems, steady state constraint, structural constraint on unstable weight

\section{Introduction}

When an MIMO plant has zeros at $s=0$ such as in 5), $6)$, it is well known that this imposes a constraint on the structure of unstable dynamics of the controller. In this note, we revisit this issue from a new standpoint, i.e. to expose the constraint on the steady state output of the plant due to this unstable zero. This, in tracking problems, implies that the reference is not free and is subject to a structural constraint. This study is necessary in the servo design using unstable weight. It will be shown that the unstable weight must also satisfy a structural constraint in this case.

We also propose a structure of generalized plant for optimal design. The Extended $\mathcal{H}_{\infty}$ control $^{3), 4)}$ can be applied to design a controller for asymptotic rejection of step disturbance or reference tracking.

The notation $G_{y x}$ denotes the transfer matrix $x \mapsto y$.

\section{Plant zero and steady state output constraint}

Let $P(s)$ be an $n$th order plant of dimension $n_{u} \times n_{u}$. Suppose a stabilizable and detectable realization is

$$
P(s)=\left[\begin{array}{c|c}
A_{p} & B_{p} \\
\hline C_{p} & 0
\end{array}\right] .
$$

Without loss of generality, it can be assumed that $B_{p}$ has full column rank and $C_{p}$ has full row rank. The plant has $n_{z}$ zeros at $s=0, n_{u}>n_{z} \geq 1$. So there exist a full row rank matrix $V=\left[\begin{array}{cc}V_{1} & V_{2}\end{array}\right], \operatorname{rank}(V)=n_{z}$, that satisfies the equation below.

\footnotetext{
${ }^{*}$ Faculty of Engineering, University of Chiba, Inage-ku, Chiba

** Faculty of Engineering, Tokyo Institute of Technology, Meguro-ku, Tokyo

(Received June 27, 1997)

(Revised February 5, 1998)
}

$$
\left(n_{z}\right) \quad\left[\begin{array}{c}
\left(n_{u}\right) \\
V_{1}, V_{2}
\end{array}\right]\left[\begin{array}{c}
A_{p} \\
-\frac{B_{p}}{C_{p}}-\frac{\overline{0}}{0}
\end{array}\right]=0
$$

Due to the stabilizability of $\left(A_{p}, B_{p}\right), V_{2}$ must be full row rank.

Assumption 1. The reference input and/or persistent disturbance are step signals.

Proposition 1. For the plant given above, the following statements hold.

(i) the steady state output $y(\infty)$ of the plant must satisfy the constraint

$$
V_{2} \cdot y(\infty)=0
$$

when $P(s)$ is stabilized by feedback control.

(ii) The step reference input $r$ can be tracked asymptotically only if $r \in \operatorname{Ker} V_{2}$.

(iii) The step output disturbance $d$ can be rejected asymptotically only if $d \in \operatorname{Ker} V_{2}$.

Proof. (i) Owing to Assumption 1, it can be proved that $\dot{x}_{p}(\infty)=0$ holds in steady state so that

$$
\left\{\begin{array}{l}
0=A_{p} x_{p}(\infty)+B_{p} u(\infty) \\
y(\infty)=C_{p} x_{p}(\infty)
\end{array}\right.
$$

By (2) we have

$$
\begin{aligned}
& {\left[\begin{array}{ll}
V_{1} & V_{2}
\end{array}\right]\left[\begin{array}{ll}
0 & y(\infty)^{T}
\end{array}\right]^{T}} \\
& =\left[\begin{array}{ll}
V_{1} & V_{2}
\end{array}\right]\left[\begin{array}{cc}
A_{p} & B_{p} \\
C_{p} & 0
\end{array}\right]\left[\begin{array}{c}
x_{p}(\infty) \\
u(\infty)
\end{array}\right] \\
& =0 .
\end{aligned}
$$

This leads to $V_{2} \cdot y(\infty)=0$.

(ii) Since $y(\infty)=r$, it follows from (3) that $V_{2} r=$ $V_{2} y(\infty)=0$.

(iii) The disturbance is added to the output, so $y(t)=$ $C_{p} x(t)+d$. Then, following the proof of $(i)$, we obtain $V_{2} y(\infty)=V_{2} d$. Therefore, $y(\infty)=0$ is possible only if $V_{2} d=0$. 


\section{Servo design using unstable weight}

Let us consider the asymptotic rejection of step output disturbance of $P(s)$. For reference tracking problem, just take the tracking error as the plant output and the rest is the same. A useful way of doing this in the framework of optimal control is to use unstable weight at output $y$, as $W_{S}$ shown in Fig. 1.

$W_{S}$ of (5) is the general form for a transfer matrix having integrator dynamics only, and $T_{W}$ is a design parameter and is to be determined. If fine tuning is required, extra stable weight can be added behind $W_{S}$ and the results of this note are not affected.

$$
W_{S}=\frac{1}{s} \times T_{W}=\left[\begin{array}{c|c}
0_{n_{1}} & T_{W} \\
\hline I_{n_{1}} & 0
\end{array}\right] .
$$

Assumption 2. $T_{W}$ has full rank.

Note at this stage, it is not clear whether $T_{W}$ should have full row rank or full column rank and how many rows $T_{W}$ should have. This is to be determined so that a control system that rejects disturbance asymptotically can be synthesized by using the unstable weight.

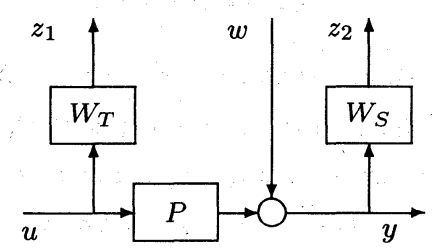

Fig. 1 Generalized plant structure

\section{Determination of $T_{W}$}

\section{1 Main result}

The key idea of using unstable weight in the design is to ensure asymptotic disturbance rejection by comprehensive stability ${ }^{1), 2)}$. That is, to guarantee $y(\infty)=0$ by $G_{z w}(s) \in \mathcal{R} \mathcal{H}_{\infty}$. The following proposition gives the requirement on the unstable weight in order to realize this purpose.

Proposition 2. Let $T_{W}$ be an $n_{1} \times n_{u}$ constant matrix and suppose there exists a controller $K(s)$ stabilizing $P(s)$ internally. Let $V_{y}$ be the matrix in equation (6). Further, let $y(t)$ be the response of $P(s)$ to the step output disturbance $d \in \operatorname{Ker} V_{2}$. Then the statements $(i)$ and (ii) below are valid.

(i) To guarantee $y(\infty)=0$ by $G_{z_{2} w}(s) \in \mathcal{R} \mathcal{H}_{\infty}, V_{y}$ must be nonsingular.

(ii) If $G_{z_{2} w}(s) \in \mathcal{R} \mathcal{H}_{\infty}$ and $V_{y}$ is nonsingular, then $y(\infty)=0$.

$$
V_{y}=\left[\begin{array}{c}
\left(n_{u}\right) \\
V_{2} \\
T_{W}
\end{array}\right] \begin{aligned}
& \left(n_{z}\right) \\
& \left(n_{1}\right)
\end{aligned}
$$

Proof. In both (i) and (ii) it is assumed that $G_{z_{2} w}(s) \in \mathcal{R} \mathcal{H}_{\infty}$. So, we investigate the condition for this. As the closed loop system $(P, K)$ is internally stable, the generalized plant of $P$ with respect to exogenous input $w$ and controlled output $z_{2}$ (Fig. 1) must be comprehensively stabilizable ${ }^{1), 2)}$. One of the comprehensive stabilizability condition requires that the unstable poles of $W_{S}$, which are not observable from $y$, be controllable from $u$. The transfer matrix $u \mapsto z_{2}$ has a realization as below

$$
W_{S} P(s)=\left[\begin{array}{cc|c}
A_{p} & 0 & B_{p} \\
T_{W} C_{p} & 0_{n_{1}} & 0 \\
\hline 0 & I_{n_{1}} & 0
\end{array}\right]
$$

We need only to find the controllability condition of this realization at $s=0$. This requires

$$
\operatorname{rank} J(0)=n+n_{1}
$$

where

$$
J(0):=\left[\begin{array}{cc:c}
A_{p} & 0 & B_{p} \\
T_{W} C_{p} & 0 & 0
\end{array}\right] .
$$

Suppose $\exists \eta=\left[\begin{array}{ll}\eta_{1} & \eta_{2}\end{array}\right] \neq 0$ such that $\eta V_{y}=0$, i.e.

$$
\eta_{1} V_{2}=-\eta_{2} T_{W}
$$

Obviously $\eta_{2} \neq 0$ since otherwise $\eta_{1}=0$ would follow from $\eta_{1} V_{2}=0$, which contradicts the assumption $\eta \neq 0$. So we have

$$
\begin{aligned}
& {\left[\begin{array}{ll}
\eta_{1} V_{1} & -\eta_{2}
\end{array}\right] J(0)} \\
& =\left[\begin{array}{ll}
\eta_{1} V_{1} & \eta_{1} V_{2}
\end{array}\right]\left[\begin{array}{ccc}
A_{P} & 0 & B_{P} \\
C_{p} & 0 & 0
\end{array}\right] \\
& =0 .
\end{aligned}
$$

This means that if $V_{y}$ does not have full row rank, at least a pole of $W_{S}$ at $s=0$ is not controllable. Therefore, $G_{z_{2} w}(s) \in \mathcal{R} \mathcal{H}_{\infty}$ is possible only if $V_{y}$ has full row rank.

Now, suppose $G_{z_{2} w}(s) \in \mathcal{R} \mathcal{H}_{\infty}$. Since

$$
G_{z_{2} w}(s)=\frac{1}{s} T_{W} G_{y w}=T_{W} G_{y w} \times \frac{1}{s} I_{n_{u}},
$$

the response $y(t)$ of any output step disturbance vector satisfies

$$
T_{W} \cdot y(\infty)=0
$$

in steady state. According to proposition $1, V_{2} y(\infty)=$ $V_{2} d=0$. So

$$
\left[\begin{array}{c}
V_{2} \\
T_{W}
\end{array}\right] \cdot y(\infty)=0
$$


holds. Then, $y(\infty)=0$ is guaranteed only if $V_{y}$ has full column rank. This proves $(i)$.

Suppose $G_{z_{2} w}(s) \in \mathcal{R} \mathcal{H}_{\infty}$. Then, it has been already shown in the first part of proof that (12) holds. Since $V_{y}$ is nonsingular, $y(\infty)=0$ follows immediately. This proves $(i i)$.

Remark 1. As $V_{y}$ is nonsingular, it follows that the number of rows of $T_{W}$ must be $n_{1}=n_{u}-n_{z}$.

Remark 2. In the controller design, a good starting point to tune $T_{W}$ is to choose it satisfying the relation $T_{W} \perp V_{2}$.

\subsection{A generalized plant}

If other specifications are required, one needs only to add extra exogenous input and controlled output to the plant $P$. An example of mixed sensitivity problem is shown in Fig. 1, where the case of plant having additive perturbation is considered. The stable weight $W_{T}=\left\{A_{T}, B_{T}, C_{T}, D_{T}\right\}$ is used to model the bound of additive perturbations. The realization of the generalized plant of Fig. 1 is given by

$$
\begin{aligned}
& G=\left[\begin{array}{c|ccc}
A & B_{1} & B_{2} \\
\hline C_{1} & D_{11} & D_{12} \\
\hdashline C_{2} & D_{21} & 0
\end{array}\right] \\
& =\left[\begin{array}{ccc|c:c}
A_{p} & 0 & 0 & 0 & B_{p} \\
0 & A_{T} & 0 & 0 & B_{T} \\
T_{W} C_{p} & 0 & 0_{n_{1}} & T_{W} & 0 \\
\hline 0 & C_{T} & 0 & 0 & D_{T} \\
0 & 0 & I_{n_{1}} & 0 & 0 \\
\hdashline C_{p} & 0 & 0 & -\frac{1}{I} & 0
\end{array}\right] .
\end{aligned}
$$

The comprehensive stabilizability of this generalized plant requires that ${ }^{1), 2)}$ :

A1) There should exist no eigenvalues of $A$ both uncontrollable from $u$ and unobservable from $y$.

A2) Among the unstable eigenvalues of $A$, those uncontrollable from $u$ (resp. unobservable form $y$ ) should be invariant zeros of $G_{12}$ (resp. $G_{21}$ )

A3) $\left[\begin{array}{ll}B_{2}^{T} & D_{12}^{T}\end{array}\right]^{T}$ should be full column rank and $\left[\begin{array}{ll}C_{2} & D_{21}\end{array}\right]$ should be full row rank.

This is verified below.

A1) The only unstable and unobservable modes of $\left(C_{2}, A\right)$ are eigenvalues of $A_{S}=0_{n_{1}}$, which are controllable in $\left(A, B_{2}\right)$ if $T_{W}$ is chosen as in Proposition 2.

A2) Since for $Y_{1}^{T}=\left[\begin{array}{lll}0 & 0 & -I\end{array}\right], Y_{2}^{T}=T_{W}$

$$
\left[\begin{array}{cc}
Y_{1}^{T} & Y_{2}^{T}
\end{array}\right]\left[\begin{array}{cc}
A & B_{1} \\
C_{2} & D_{21}
\end{array}\right]=A_{S}\left[\begin{array}{cc}
Y_{1}^{T} & 0
\end{array}\right]
$$

holds, the eigenvalues of $A_{S}$ are invariant zeros of $G_{21}=\left\{A, B_{1}, C_{2}, D_{21}\right\}$.
A3) Since $B_{p}$ has full column rank and $C_{p}$ has full row rank, this is automatically satisfied.

\section{Example}

Consider the plant below.

$$
P=\left[\begin{array}{ccc|cc}
-\tau_{1} & -k_{t 1} & 0 & k_{t 1} & 0 \\
k_{1} & 0 & -k_{2} & 0 & 0 \\
0 & -k_{t 2} & -\tau_{2} & 0 & k_{t 1} \\
\hline 1 & 0 & 0 & 0 & 0 \\
0 & 0 & 1 & 0 & 0
\end{array}\right]
$$

This plant has $n_{z}=1$ zero at $s=0$ and the zero vector is

$$
\left.V=\left[V_{1}{ }^{\prime}, V_{2}\right]=\left[\begin{array}{lllll}
0 & 1 & 0 &
\end{array}\right]-k_{1} \quad k_{2}\right] .
$$

Following Proposition 2 and Remark 2, we choose $T_{W}$ as

$$
T_{W}=\left[\begin{array}{ll}
k_{2} & k_{1}
\end{array}\right]
$$

So, the output weighting function is given by

$$
W_{S}=\left[\begin{array}{c|cc}
0 & k_{2} & k_{1} \\
\hline 1 & 0 & 0
\end{array}\right]
$$

\section{Conclusion}

We have shown that the zeros at $s=0$ imposes a constraint on the steady state output of the plant. Due to the existence of this zero, the output unstable weight used in the servo synthesis must also be subject to a structural constraint. The constraint on the output unstable weight is also exposed.

If the constraint on the output weighting function is satisfied, one can use Extended $\mathcal{H}_{\infty}$ control theory to design a controller achieving asymptotic tracking and/or disturbance rejection.

\section{References}

1) K. Z. Liu, T. Mita: A unified stability analysis for regulator and servomechanism problems, Proceedings of the 33rd CDC, 4198 / 4203 (1994).

2) 劉 康志, 美多勉: 線形レギュレータとサーボ問題に対する統一 安定解析, 計測自動制御学会論文集, 31-7, 834 / 843 (1995).

3) T. Mita, K. Kuriyama, K. Z. Liu: $\mathcal{H}_{\infty}$ Control with unstable weighting functions - a design method of $\mathcal{H}_{\infty}$ robust servo systems, Proceedings of 32nd CDC, 650 / 655 (1993).

4) 美多勉, 劉 康志, 栗山: 虚軸上に極を持つ重みを許す $\mathcal{H}_{\infty}$ 制 御系の設計, 計測自動制御学会論文集, 29-11, 1320 / 1329 (1993).

5) S. B. Villas-Boas, T. Mita, K. Z. Liu: Extended $\mathcal{H}_{\infty}$ Controller Applied to Frequency Control of High-Power Generation System, Proceedings of 35th CDC, 761 / 766 (1996).

6) S. B. Villas-Boas, T. Mita, K. Z. Liu: Application of Extended $\mathcal{H}_{\infty}$ Control to Multi Area Frequency Control of Power Generation System, システム制御情報学会誌, 11-4, 182 / 189 (1998). 